This document is confidential and is proprietary to the American Chemical Society and its authors. Do not copy or disclose without written permission. If you have received this item in error, notify the sender and delete all copies.

\title{
When Ellipsometry Works Best - A Case Study With Transparent Conductive Oxides
}

\begin{tabular}{|r|l|}
\hline Journal: & ACS Photonics \\
\hline Manuscript ID & ph-2020-00389j.R2 \\
\hline Manuscript Type: & Article \\
\hline Dute Submitted by the & n/a \\
\hline Complete List of Authors: & $\begin{array}{l}\text { Hillier, James; Nottingham Trent University, School of Science and } \\
\text { Technology } \\
\text { Camelio, Sophie; Université de Poitiers, Institut Pprime } \\
\text { Cranton, Wayne; Sheffield Hallam University, Materials and Engineering } \\
\text { Research Institute } \\
\text { Nabok, Alexei; Sheffield Hallam University, Materials and Engineering } \\
\text { Research Institute } \\
\text { Mellor, Christopher; University of Nottingham, School of Physics and } \\
\text { Astronomy } \\
\text { C. Koutsogeorgis, Demosthenes ; Nottingham Trent University, School of } \\
\text { Science \& Technology } \\
\text { Kalfagiannis, Nikolaos; Nottingham Trent University, School of Science } \\
\text { and Technology }\end{array}$ \\
\hline
\end{tabular}

\section{SCHOLARONE Manuscripts}




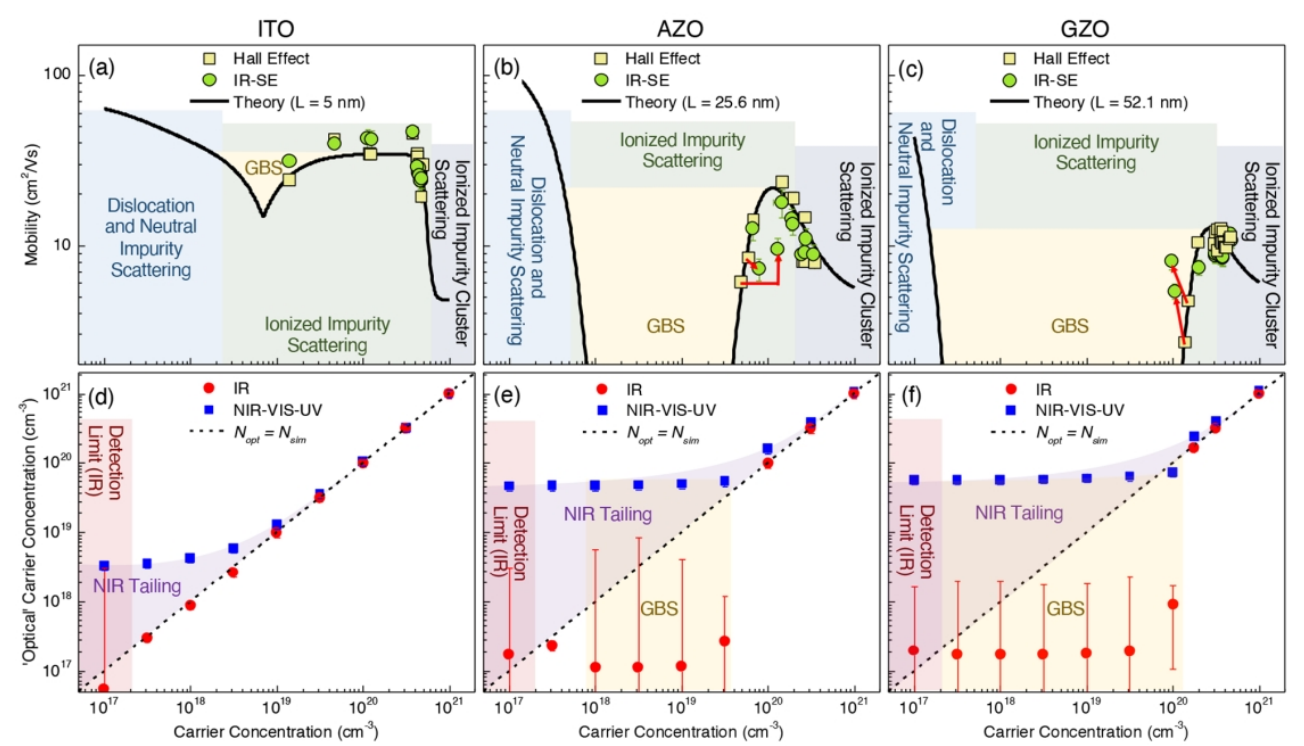

Figure 1. (a-c) Carrier concentration and mobility of sputtered ITO, AZO and GZO films, respectively, as determined by IR-SE (green circles) and Hall Effect (yellow squares). The fit of $\mu(N)$ (eqs 2-7) to the Hall Effect measurements is shown by the black solid line. We highlight the regions where different scattering mechanisms are dominant with the different colors. (d-f) 'Optical' carrier concentration, $N_{o p t}$, as determined

by fitting each simulated dataset of $N_{\text {sim }}$ for ITO, AZO and GZO, respectively. We show the result from fitting the data simulated in the IR (red circles) and in the NIR-VIS-UV (blue squares) alongside $N_{\text {opt }}=N_{\text {sim }}$ (black dashed diagonal line). The influence of the NIR tailing and GBS are indicated by the purple and yellow shaded areas, respectively.

$170 \times 96 \mathrm{~mm}(300 \times 300 \mathrm{DPI})$ 



Figure 2. (a-c) Real, $\varepsilon_{1}(E)$, and (d-f) imaginary, $\varepsilon_{2}(E)$, parts of the complex permittivity for the sputtered ITO, AZO and GZO films, respectively, as fit in the IR (solid red lines), as fit in the NIR-VIS-UV (solid blue lines), via extrapolation of the NIR-VIS-UV into the IR (dashed blue lines), and as simulated with only the Drude term of the IR fitted (dashed black/grey lines). 'Low- $N$ ' (darker lines) and 'High- $N$ ' (lighter lines) represent two distinct films of each material which cover the experimental range of carrier concentration. The purple shaded region highlights the difference between the simulated and fitted Drude contribution in the NIR, where tailing effects of the IR absorption centers are mistaken for free carrier absorption when fitting in the NIR-VIS-UV. Please note the changes in scale at -17 and 7 for the real permittivity and at 3 for the imaginary permittivity.

$170 \times 90 \mathrm{~mm}(300 \times 300 \mathrm{DPI})$ 


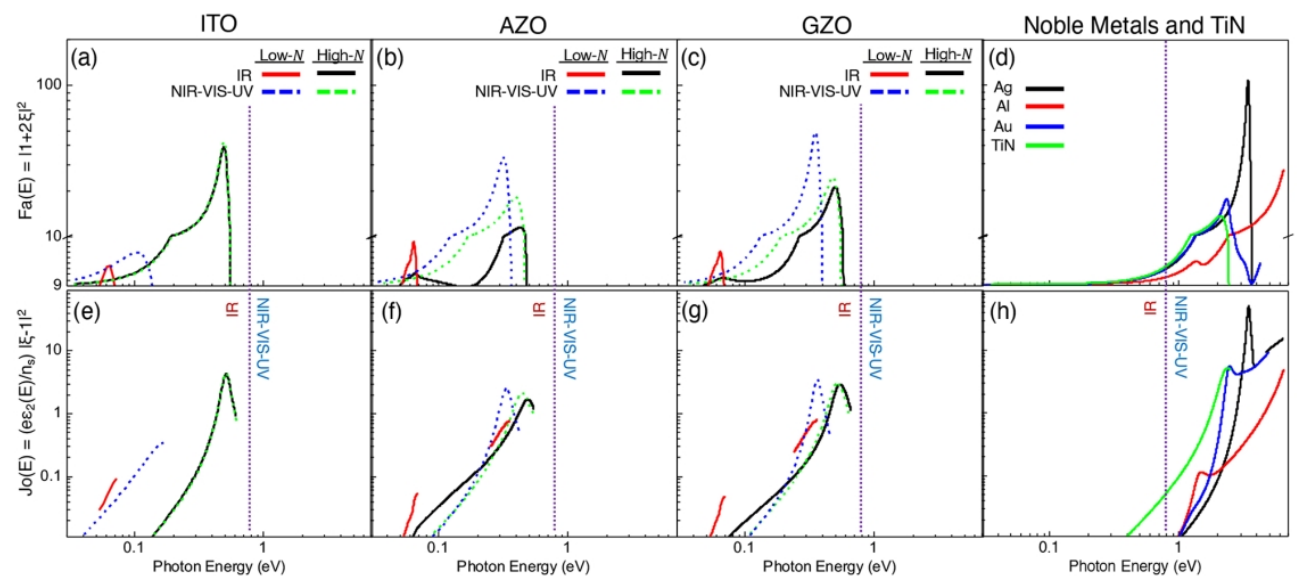

(a-d) Faraday and (e-h) Joule numbers for ITO, AZO and GZO, respectively, alongside (d,h) Ag (black), Al (red), Au (blue) and TiN (green) for comparison. For the TCOs, we present FoMs for the high-N (black solid and green dashed lines) and low- $N$ (red solid and blue dashed lines) films. The solid lines represent the 'true' FoM (calculated by optical constants derived by IR-SE) taking into account phonon and interband transitions and the dashed lines are the calculations employing extrapolated optical constants from NIR-VISUV-SE. Please note the logarithmic scales and the change in scale in the Faraday number at 10.

$170 \times 77 \mathrm{~mm}(300 \times 300 \mathrm{DPI})$ 


\title{
When Ellipsometry Works Best - A Case Study
}

\section{With Transparent Conductive Oxides.}

\author{
James A. Hillier, Sophie Camelio, Wayne Cranton, Alexei V. Nabok, Christopher J. Mellor, \\ Demosthenes C. Koutsogeorgis, and Nikolaos Kalfagiannis*
}

KEYWORDS. Transparent Conductive Oxides, Spectroscopic Ellipsometry, Nano-photonics, Infrared Plasmonics, Dielectric function, Figures of Merit.

\begin{abstract}
As the library of potential materials with plasmonic behavior in the infrared (IR) grows, we must carefully assess their suitability for nano-photonic applications. This assessment relies on knowledge of the materials' optical constants, best determined via spectroscopic ellipsometry (SE). Transparent conductive oxides are great candidates for IR plasmonics due to their low carrier concentrations (compared to noble metals) and the ability to tailor their carrier concentration by manipulating the defect composition. When the carrier concentration becomes low enough, phonon and defect states become the dominant mechanism of absorption in the IR spectral range, leading to near-IR (NIR) tailing effects. These NIR tailing effects can be misinterpreted for free carrier absorption, rendering NIR-visible-ultraviolet-SE (NIR-VIS-UV-SE) incapable of reliably extracting the carrier transport properties. In this work, we report the limitations of NIR-VIS-UV and IR-SE (in terms of carrier concentration) by investigating the transport mechanisms of indium
\end{abstract}


tin oxide, aluminium-doped zinc oxide and gallium-doped zinc oxide. We find regions of carrier concentration where NIR-VIS-UV-SE cannot reliably determine the transport properties and we designate material-dependent and application-specific confidence factors for NIR-VIS-UV-SE. For IR-SE, the story is more complex and so we investigate the multi-faceted influences on the limitations, such as phonon behavior, grain size, presence of a substrate, film thickness and measurement noise. Finally, we demonstrate the importance of identifying the IR optical constants directly via IR-SE (rather than by extrapolation from NIR-VIS-UV-SE) by means of-comparing specific figures of merits (Faraday and Joule numbers), deemed useful indicators for plasmonic performance.

The promise of plasmonics is to usher in a new generation of opto-electronic devices through the exploitation of the strong interactions between light and free electrons at the nanoscale. ${ }^{1,2}$ The free electrons oscillate in response to the electric field, giving rise to surface plasmon polaritons (SPP) in planar metal/dielectric interfaces. Modern nanofabrication techniques have the ability to create metallic nanostructures which can confine light within dimensions far below the wavelength of light via excitation of localized surface plasmon resonances (LSPR). ${ }^{3}$ By manipulating the nanoarchitecture, a wide variety of plasmonic responses have been demonstrated. Such architectures were able to advance applications in nanophotonics, ${ }^{4-6}$ solar energy harvesting ${ }^{7,8}$ biosensing,,${ }^{9,10}$ optical encoding, ${ }^{11}$ photo-catalysis, ${ }^{12,13}$ waveguiding ${ }^{14}$ as well as the most impactful area of cancer treatment. ${ }^{15,16}$

The bulk of research into plasmonic device design has focused on the plethora of potential nanoarchitectures that can be utilized either in order to enhance the plasmonic performance for 
particular applications or to tune the operational window towards unexplored spectral ranges. So far, the majority of research works utilize $\mathrm{Au}$ and $\mathrm{Ag}$, which produce the strongest resonances due to their unrivalled conductivity. ${ }^{17,18}$ However, this route has stalled the translation of these concepts into real and practical devices due to certain limitations of the noble metals, i.e. their incompatibility with CMOS techniques ${ }^{19}$ and their low-melting-point and high diffusivity (preventing their use at high-temperatures). Another key factor is that mono-atomic metals cannot be doped. Alloys can be made of different metals in order to tune the plasmon resonance, but such processes have limitations (i.e. ultrahigh doping challenges, interband transitions and degraded relaxation times). ${ }^{20-23}$ As such, the operating frequency of a plasmonic nanostructure is generally tuned by varying the geometrical features (shape and size) as well as changing the local dielectric environment. This often causes compromises in their performance due to increased electronic losses at longer wavelengths.

Recently, the focus has shifted towards alternative materials which overcome these limitations, including refractory metals, ${ }^{24}$ transition metal nitrides, ${ }^{25,26}$ and transparent conductive oxides (TCOs) such as tin-doped indium oxide (ITO), aluminium-doped zinc oxide (AZO) and galliumdoped zinc oxide (GZO). ${ }^{27-29}$ The quest for alternative materials stems from the desire to expand the operational frequency without sacrificing performance. For example, extension of the resonance into the infrared (IR) can enable high-sensitivity label-free detection of molecules via vibrational/rotational spectroscopy. ${ }^{17}$ At far IR and terahertz $(\mathrm{THz})$ wavelengths, plasmonics has been proposed to benefit applications for photothermal detection, $\mathrm{THz}$ sources and imaging through opaque objects. ${ }^{30}$ Vitally, the non-retarded frequency for plasmon resonance of nitrides and TCOs can be tuned through the manipulation of the defect composition ${ }^{31,32}$ and application of an electric field via the Pockels effect. ${ }^{33}$ 
Materials with carrier concentrations below $\sim 10^{21} \mathrm{~cm}^{-3}$, such as TCOs, are appealing as active plasmonic elements in the IR regime. To assess their prospects, we require precise knowledge of the optical constants, taking into account the influence of optical phonons and/or defect states. Uprety and colleagues ${ }^{34,35}$ previously demonstrated how IR spectroscopic ellipsometry (SE) is vital for gaining such knowledge. By simultaneously fitting SE data of ITO and AZO in the nearIR-visible-ultraviolet (NIR-VIS-UV) $(0.75-5.89 \mathrm{eV})$, IR $(0.035-0.4 \mathrm{eV})$ and THz $(0.3-4.1 \mathrm{meV})$ regimes, they demonstrated how the NIR absorption should be interpreted as a combination of tailing effects from free carriers, phonons and/or low-energy defect states. They reported that fitting the NIR-VIS-UV resulted in overestimating the contribution of free carriers and that extension of the measured spectral range into the IR and $\mathrm{THz}$ regions produced greater agreement with 4-point probe measurements. In addition, Wang et al. ${ }^{36}$ highlighted the requirement for extended frequency ranges to understand the conductivity of TCOs by noting how the presence of phonon modes in doped $\mathrm{ZnO}$ and ITO affect the measurement of the free electron relaxation.

Despite these findings, a large proportion of SE-based investigations of metal oxides still rely on SE in the NIR-VIS-UV and the use of IR-SE remains quite rare. ${ }^{27,37-41}$ This reliance leads to false-positive results when it comes to metal oxides and other low carrier concentration materials, such as doped semiconductors. In this work, we examine a library of ITO, AZO and GZO sputtered films in order to assess the relationship between the carrier mobility and the carrier concentration elucidating the electron transport mechanisms (which define both plasmonic and electronic performance) via the different scattering regimes and non-parabolicity in the conduction band. ${ }^{42-}$ ${ }^{45}$ The knowledge generated in the present study expands further the findings of Uprety and colleagues $^{34,35}$ and Wang et al. ${ }^{36}$ We demonstrate when IR-SE measurements become necessary for materials such as metal oxides. We find that NIR tailing causes NIR-VIS-UV-SE to become 
increasingly ambiguous for metal oxides with carrier concentrations within a 'region of uncertainty'. We tie the upper boundary of the region of uncertainty to an application-specific 'acceptable deviation' in the carrier concentration. Due to the sensitivity of plasmonic properties to the carrier concentration, in this work, we apply an acceptable deviation of $10 \%$ and we find that this region exists below $(3.0 \pm 0.5) \times 10^{19} \mathrm{~cm}^{-3},(7 \pm 1) \times 10^{20} \mathrm{~cm}^{-3}$ and $(7 \pm 2) \times 10^{20}$ $\mathrm{cm}^{-3}$ for ITO, AZO and GZO, respectively. We go further by considering the effects of inherent material limitations, noise and the presence of a substrate, across a wide range of carrier concentrations, in order to further elucidate the more complex limits that are encountered by IRSE. Additionally, by applying this knowledge we are able to present figures of merit (FoM) for two key potential plasmonic applications in the IR, namely the near-field enhancement and photothermal conversion, for ITO, AZO and GZO at the, currently un-reported, IR frequencies. This work will incentivize a reconsideration of strategies to unveil the origins of the features of the complex permittivity of alternative materials for IR plasmonics, such as oxides, nitrides and doped semiconductors.

\section{RESULTS AND DISCUSSION \\ EXPERIMENTAL INVESTIGATION}

A set of ITO, AZO and GZO thin films were fabricated via room temperature magnetron sputtering on top of test-grade n-type $\mathrm{Si}$ wafers (resistivity range: $1-20 \Omega \mathrm{cm}$ ). For each material, the deposition conditions were varied in order to produce films with similar crystal structure while covering a range of carrier concentrations. Further details on the experimental procedure and fabrication conditions can be found in the supporting information (SI), Section 1.1. The permittivity of each thin film was characterized via IR-SE. The permittivity is described by eq 1 
as a summation of the high-frequency permittivity, $\varepsilon_{\infty}$, a Drude term (describing free carrier absorption) and additional individual oscillators, $\tilde{\varepsilon}_{n \cdot}{ }^{46}$

$$
\tilde{\varepsilon}(E)=\varepsilon_{\infty}-\frac{\hbar^{2}}{\varepsilon_{o} \rho_{o p t}\left(\tau_{o p t} E^{2}+i \hbar E\right)}+\sum_{n=1}^{m} \tilde{\varepsilon}_{n}(E)
$$

where $\hbar$ is the reduced Planck's constant, $E$ is the photon energy, $\varepsilon_{0}$ is the vacuum permittivity, $\rho_{\text {opt }}$ is the 'optical' resistivity and $\tau_{\text {opt }}$ is the 'optical' carrier mean free time. The third term represents a sum of $m$ oscillators that describe phonon, defect state and interband absorption.

Further details of the SE equipment, fitting methodology and the individual oscillator functions can be found in the SI, Section 1.4. We follow the approach of Fujiwara et al., ${ }^{44}$ and others, ${ }^{42,43,45}$ to consider the change in effective electron mass, $m_{e}^{*}$, with the carrier concentration, $N$, by taking into account the non-parabolicity of the conduction band as it is populated with free carriers. We do this by performing Hall Effect measurements which, when combined with IR-SE, allow us to directly calculate $m_{e}^{*}$ for each sputtered thin film and determine how $m_{e}^{*}$ varies with $N, m_{e}^{*}(N)$, for ITO, AZO and GZO. The $m_{e}^{*}(N)$ relationship is then utilized to calculate the carrier concentration and mobility, $\mu$. This process is described in detail in the SI, Section 3. Figures 1ac present the optical (green circles) and Hall (yellow squares) carrier concentration and mobility of the sputtered ITO, AZO and GZO films, respectively. The relationship between $\mu$ and $N$ follows the trend described by Ellmer et al. ${ }^{47}$ The $\mu(N)$ relationship is calculated for each material by following eqs 2-7 presented below. This approach accounts for the effects of grain boundary scattering (GBS) (via Seto's model) ${ }^{48}$ and ionized impurity scattering (IIS) (via Masetti’s model) ${ }^{49}$ and ignores both dislocation scattering and neutral impurity scattering.

$$
\frac{1}{\mu(N)}=\frac{1}{\mu_{I I S}(N)}+\frac{1}{\mu_{G B S}(N)}
$$


where:

$$
\begin{aligned}
& \mu_{I I S}(N)=\mu_{\min }+\frac{\mu_{\max }-\mu_{\min }}{1+\left(\frac{N}{N_{\text {ref } 1}}\right)^{\alpha_{1}}}-\frac{\mu_{1}}{1+\left(\frac{N_{r e f 2}}{N}\right)^{\alpha_{2}}} \\
& \mu_{G B S}(N)=\mu_{0}(N) \cdot \exp \left(-\frac{E_{b}(N)}{k_{b} T}\right)
\end{aligned}
$$

where $k_{b}$ and $T$ are the Boltzmann constant and temperature, respectively. $\mu_{\max }$ is indicative of the lattice mobility, $\mu_{\min }$ the ionised impurity inhibited mobility and $\mu_{\min }-\mu_{1}$ of the ionised cluster inhibited mobility. $N_{r e f 1}$ and $\alpha_{1}$, are the transition concentration and coefficient, respectively, for ionized impurities while $N_{r e f 2}$ and $\alpha_{2}$ are the transition concentration and coefficient, respectively, for clusters of ionized impurities. $E_{b}(N)$ is the grain boundary height, for which the formulation changes depending on whether the grain is fully depleted and the traps are partially filled (eq 5), or the grain is partially depleted while the traps are fully filled (eq 6). ${ }^{48}$

$$
\begin{gathered}
E_{b}(N)=\frac{e^{2} Q_{t}^{2}}{8 \varepsilon_{r} \varepsilon_{0} N}, \text { for } L N>Q_{t} \\
E_{b}(N)=\frac{e^{2} L^{2} N}{8 \varepsilon_{r} \varepsilon_{0}}, \text { for } L N<Q_{t} \\
\mu_{0}(N)=\frac{e L}{\sqrt{2 \pi \cdot m_{e}^{*}(N) \cdot k_{b} T}}
\end{gathered}
$$

where $\varepsilon_{0}, \varepsilon_{r}, e, L$ and $Q_{t}$ are the vacuum permittivity, static dielectric constant, elementary charge, grain size and trap density, respectively.

The $\mu(N)$ relationship, as fitted to Hall Effect measurements of the sputtered TCOs, is demonstrated with the black solid lines in Figure 1a-c. When fitting $\mu(N)$ to the experimental data only the parameters $N_{r e f 2}$ and $Q_{t}$ were left free, for AZO and GZO, with an additional requirement for $\alpha_{2}$ as a free parameter for ITO. The parameters which influence $\mu(N)$ outside the experimental 
range were taken from Ellmer et al., ${ }^{50}$ where the experimental points covered a wider range of carrier concentrations. The input of grain size into eqs 6-7 was obtained via X-ray diffraction (see SI, Section 1.3). Table 1, below, outlines the parameters of $\mu(N)$ for each material.

Table 1. Fixed and free parameters of $\mu(N)$ for ITO, AZO and GZO.

\begin{tabular}{|c|c|c|c|c|}
\hline \multirow{2}{*}{$\begin{array}{l}\text { Fixed } \\
\text { Parameters }\end{array}$} & \multirow{2}{*}{ Unit } & \multicolumn{3}{|l|}{ Material } \\
\hline & & ITO & AZO & GZO \\
\hline$\mu_{\max }$ & $\mathrm{cm}^{2} / V s$ & 210 & 210 & 210 \\
\hline$\mu_{\min }$ & $\mathrm{cm}^{2} / V s$ & 55 & 55 & 55 \\
\hline$\mu_{\min }-\mu_{1}$ & $\mathrm{~cm}^{2} / V s$ & 5 & 5 & 5 \\
\hline$N_{r e f 1}$ & $\times 10^{17} \mathrm{~cm}^{-3}$ & 15 & 4 & 4 \\
\hline$\alpha_{1}$ & & 1 & 1 & 1 \\
\hline$\alpha_{2}$ & & -- & 2 & 2 \\
\hline$L$ & $n m$ & 5 & 25.6 & 52.1 \\
\hline$\varepsilon_{r}$ & & 9 & 8.3 & 8.3 \\
\hline$m_{0}^{*}$ & & $0.21 \pm 0.02$ & $0.24 \pm 0.03$ & $0.26 \pm 0.02$ \\
\hline$C$ & & $0.4 \pm 0.1$ & $0.6 \pm 0.2$ & $0.6 \pm 0.1$ \\
\hline Free & \multirow{2}{*}{ Unit } & \multicolumn{3}{|l|}{ Material } \\
\hline Parameters & & ITO & AZO & GZO \\
\hline$N_{r e f 2}$ & $\times 10^{20} \mathrm{~cm}^{-3}$ & $5.1 \pm 0.3$ & $1.2 \pm 0.1$ & $1.50 \pm 0.05$ \\
\hline$\alpha_{2}$ & & $14 \pm 10$ & -- & -- \\
\hline$Q_{t}$ & $\times 10^{12} \mathrm{~cm}^{-2}$ & $3.7 \pm 0.5$ & $14.2 \pm 0.5$ & $27.0 \pm 0.5$ \\
\hline
\end{tabular}

$m_{0}^{*}$ and $C$ describe how $m_{e}^{*}$ changes with carrier concentration. They are extracted by fitting the set of Hall Effect and SE data presented in Figure 1a-c (see SI, Section 3).

The influence of the grain size on the $\mu(N)$ relationship is seen in Figures 1a-c. We first note a unique behavior for ITO, while some similarities are palpable for AZO and GZO. The most 
noticeable deviation between each material is in the region where GBS is dominant, which vastly inhibits the carrier mobility within the 'mobility hole' region. We highlight this region with the yellow shaded area in Figures 1a-c. The boundaries of this region, with respect to $N$, result from $L$ and $Q_{t}$ (eqs 5-6).

Room temperature sputtered ITO thin films are nanocrystalline whereas sputtered AZO and GZO films follow a columnar structure with larger grains. ${ }^{39}$ As the grain size increases, so does $\mu_{0}$ (eq 7) and therefore the mobility at very low- $N$ is larger. However, concurrently, the region where GBS becomes dominant covers a larger range of carrier concentrations. This is vital to consider for plasmonic material components, as materials with carrier concentrations within the GBS region will have poor ability to exploit plasmonic responses in the IR due to their inhibited carrier mobility. The onset of GBS with regard to $N$ is defined by the trap density over the grain size, ${ }^{47,51}$ so this limit may be reduced to lower carrier concentrations by reducing the trap density. This could be achieved by varying the growth conditions and/or following a post annealing process. Another option would be to take advantage of the ability of the high conductivity of amorphous ITO and IGZO films, ${ }^{52,53}$ which negates the influence of GBS. ${ }^{48}$ This is noted from Figure 1a where, for ITO, there is no region where $\mu \approx 0 \mathrm{~cm}^{2} / V s$.

In Figures $1 \mathrm{~b}$ and $1 \mathrm{c}$, we observe few IR-SE experimental points $\left(N_{\text {opt }}=8 \pm 1 \times 10^{19} \mathrm{~cm}^{-3}\right.$ and $N_{\text {opt }}=1.3 \pm 0.2 \times 10^{20} \mathrm{~cm}^{-3}$ for AZO and $N_{\text {opt }}=1.0 \pm 0.1 \times 10^{20} \mathrm{~cm}^{-3}$ and $N_{\text {opt }}$ $=1.1 \pm 0.1 \times 10^{20} \mathrm{~cm}^{-3}$ for GZO) which deviate from the trend defined by the $\mu(N)$ relationship. Interestingly, these points do not agree with results from Hall Effect measurements (marked by red arrows in Figure 1b-c), even after corrections have been applied taking into account how $m_{e}^{*}$ varies with $N$. Evidently, they produce erroneous values for $m_{e}^{*}$ when evaluated against Hall Effect results (see SI, Section 3 ). For such points, $N_{\text {Hall }}$ lies within the region of GBS. Based 
on the above understanding, we interpret that those results are invalid due to the diminished mobility invoked by GBS. To verify where precise determination of the transport properties is possible, for both IR and NIR-VIS-UV-SE, we must already have precise knowledge of the carrier concentration and mobility. In addition, we need to go further down in carrier concentration values than the range covered by the experimental set of films in order to verify that SE becomes viable below the region of GBS. In response to these limitations, we performed the following ellipsometric theoretical experiment: we simulated the permittivity of ITO, AZO and GZO, extending the range of $N$ covered by the experimental dataset while following careful rules that preserve the physical reality of such simulated datasets.

\section{THEORETICAL INVESTIGATION}

To produce the simulated data sets of the bulk permittivity, for each material, we vary the simulated carrier concentration, $N_{\text {sim }}$, between $10^{17}-10^{21} \mathrm{~cm}^{-3}$. We calculate the simulated carrier mobility, $\mu_{\text {sim }}$, from eqs 5-10, taking into account $m_{e}^{*}(N)$, and subsequently calculate $\rho_{\text {sim }}$ and $\tau_{\text {sim. }}$. This brings the resistivity range between $10^{-4}-10^{7} \Omega \mathrm{cm}$. For each material, the contributions of IR and UV absorption are determined from fitting IR and NIR-VIS-UV-SE measurements of the film with a low carrier concentration (see SI, Section 5), so that the screening of the phonon and/or defect state absorption by the free carriers is minimized. The permittivity is built from the summation of the IR and UV oscillators of each material while the Drude term is defined by $\rho_{\text {sim }}$ and $\tau_{\text {sim }}$ for each simulated data set, as per eq 1 . These steps, alongside those applied to establish $\mu(N)$ and $m_{e}^{*}(N)$, ensured that the simulated data are self-consistent. For every increment of $N_{\text {sim }}$, two distinct data sets are simulated, reflecting the ranges defined by two individual ellipsometers $(0.034-0.8 \mathrm{eV}$ for the IR and $0.775-6.5 \mathrm{eV}$ for the NIR-VIS-UV). 
To elucidate the limitation of the capabilities of SE, in the different spectral ranges, we fit the simulated data sets using an identical model to the simulation (with all parameters left free) and record the 'optical' carrier concentration, $N_{\text {opt }}$, against $N_{\text {sim }}$. The results from fitting the NIR-VISUV (blue squares) and IR (red circles) simulated data sets for ITO, AZO and GZO are presented in Figures 1d, 1e and 1f, respectively. The dashed black lines indicate the ideal case where $N_{\text {opt }}=$ $N_{\text {sim }}$, which is valid for high enough carrier concentrations. As the carrier concentration decreases, we note three distinct areas where $N_{\text {opt }} \neq N_{\text {sim }}$. Each area is color coded in a way that we distinguish between different mechanisms. As the carrier concentration falls below a certain threshold (varied for each material) we notice that NIR-VIS-UV-SE (blue squares) deviates from the diagonal dashed line. This is indicated as the purple shaded areas in Figures 1d-f, where $N_{\text {opt }} \neq$ $N_{\text {sim }}$ is exclusively due to NIR tailing effects (a NIR permittivity that resembles free electron absorption but is, instead, the result of additional oscillators in the IR). The results from fitting the NIR-VIS-UV data sets maintain a negligible error in $N_{\text {opt }}$. This manifests a potential 'false positive' of NIR-VIS-UV-SE. At larger $N$, the free carrier absorption sufficiently screens the other contributions in the IR, ensuring a more accurate determination of the transport properties. We quantify how the competition between the free carrier absorption and other factors of NIR tailing affects the final determination of $N$ with the ratio $N_{\text {opt }} / N_{\text {sim }}$ (the ratio of NIR-tailing affected carrier concentration to the 'actual' carrier concentration). By fitting a logistic function to the set of $N_{\text {opt }} / N_{\text {sim }}$ (Figure S10a-c in SI), we can extract $N_{\text {sim }}$ at the point where $N_{\text {opt }} / N_{\text {sim }}$ is within an 'acceptable deviation'. Here, we consider an acceptable deviation to be $\leq 10 \%$. For ITO, this results in an upper boundary of $N_{\text {sim }}=(3.0 \pm 0.5) \times 10^{19} \mathrm{~cm}^{-3}$. Below this boundary exists a 'region of uncertainty' where one might have doubts on the validity of NIR-VIS-UV-SE to determine the carrier concentration. The relatively low acceptable deviation that we apply in this 
work is deemed suitable for plasmonics, as the plasmonic properties are very sensitive to the carrier concentration (see SI, Section 4.4). For other applications, however, the acceptable deviation may differ, resulting in an application-specific upper boundary of $N$ for the region of uncertainty. The acceptable $N_{\text {opt }} / N_{\text {sim }}$ ratio (or $\rho_{\text {sim }} / \rho_{\text {opt }}$ ratio) can become an adjustable parameter and thus fixed to the particular requirements of an application. For example, sheet resistances of $150-450 \Omega / \square$ are considered sufficient for flat panel display electrode applications, ${ }^{54}$ resulting in an acceptable deviation of $200 \%$. In addition, commercial grade ITO films are specified at $1-2 \times 10^{-4} \Omega \mathrm{cm}$ (a 100\% deviation). ${ }^{55}$ This provides an application-specific confidence factor for NIR-VIS-UVSE. A more extended analysis of the validity of the region of uncertainty is presented in the SI, Section 4.4.

For the data sets simulated in the IR, a deviation from the diagonal dashed line, coupled with a significantly increased error bar, is noted for $N_{\text {sim }} \approx 10^{17} \mathrm{~cm}^{-3}$. This is indicated as the red shaded areas in Figure 1d-f, where $N_{\text {opt }} \neq N_{\text {sim }}$ is due to the 'IR detection limit'. This wavelength dependent limit is due to rounding errors for simulated bulk films. For practical measurements the detection limit varies significantly due to a combination of the presence of noise (investigated by manually adjusting the noise in the data set), material/substrate reflections (investigated by simulating the film with varying thickness on a substrate) and/or IR absorption centers. Additionally, the IR detection limit can be reduced by utilizing films, substrates or optical spacers with a thickness sufficient to produce interference effects within the measured spectral range. These effects are explored in more detail in the SI, Section 4.

For AZO (Figure 1e), the influence of NIR tailing is higher than for ITO (Figure 1d) and so our simulations reveal that the upper boundary of the region of uncertainty for NIR-VIS-UV-SE (where $N_{\text {opt }} / N_{\text {sim }}=1.1$ ) is shifted to $(7 \pm 1) \times 10^{20} \mathrm{~cm}^{-3}$. The NIR tailing effects for GZO are 
near identical to those for AZO (see SI, Section 5.2) and so we report a similar region of uncertainty for when $N_{\text {sim }} \leq(7 \pm 2) \times 10^{20} \mathrm{~cm}^{-3}$ (see SI, Section 4.4). To the best of our knowledge, a falsepositive measurement of the carrier concentration of ITO via NIR-VIS-UV-SE has, as-of-yet, not been reported. This is likely because the most prominent implementation of TCOs has been electronic applications, ${ }^{38-41}$ or plasmonics at the telecom wavelengths, ${ }^{27,37}$ where larger carrier concentrations are desired. However, there is a collection of studies which extract the carrier transport properties of AZO and GZO from NIR-VIS-UV-SE or VIS-NIR reflection measurements while the carrier concentrations is close-to or within the region of uncertainty established in this work. $^{29,56-60}$ Furthermore, as the plasmonic community explores materials with lower $N$ in order to exploit IR plasmonics, the requirement for IR-SE becomes even more pronounced.

An additional case where $N_{\text {opt }} \neq N_{\text {sim }}$ arises when fitting AZO in the IR. When $N_{\text {sim }}=10^{18}-$ $10^{19.5} \mathrm{~cm}^{-3}, N_{\text {opt }}$ is considerably underestimated and the error in $N_{\text {opt }}$ is enormous (yellow shaded area in Figure 1e). This region directly overlaps the region where $\mu$ is diminished by the influence of GBS (yellow shaded area in Figure 1b). This diminished mobility produces an extremely damped Drude term which becomes undetectable within either (or in fact any) spectral windows. It is apparent that in order to determine the carrier concentration of a material via SE, the $N$ must fall outside the window of GBS. Indeed, for cases where GBS is an important scattering mechanism, the failure of SE due to GBS overrides the uncertainty introduced by NIR tailing. It should be noted that the simulation has assumed that the grain size and trap density do not change with carrier concentration. Accounting for this change would likely shift the low- $N$ boundary for GBS (see SI, Section 3). However, if this boundary is higher than the detection limit of IR-SE ( $\sim 10^{17} \mathrm{~cm}^{-3}$ in the presented cases), accurate determination of the transport properties is possible below the GBS threshold (see the point $N_{\text {sim }}=10^{17.5} \mathrm{~cm}^{-3}$ in Figure 1e). We find similar results 
for GZO, with the exception that the window of GBS is large enough to overlap with the fundamental detection limits so that there is no region of $N_{\text {opt }}=N_{\text {sim }}$ below $10^{20.5} \mathrm{~cm}^{-3}$. The above-mentioned results have a prominent effect in the complex permittivity of the studied, sputtered, metal oxides. From the variety of samples presented above (Figures 1a-c) we choose two distinct thin films of each material that cover the experimental range of $N$. We denote these samples as 'low- $N$ ' and 'high- $N$ ' hereafter and in the SI. The permittivity of these samples was determined from both IR and NIR-VIS-UV-SE measurements. Specifically, the parameters describing the film thickness, surface roughness, high-frequency permittivity, and interband transitions were determined from NIR-VIS-UV-SE and fixed during the fitting of IR-SE data. This 'fitting strategy' was deemed to provide the best agreement with alternative measurement techniques (reflectance spectroscopy, 4pp, atomic force microscopy, see SI, Section 2). The measurements, fit, graphical and parameterized permittivity of these samples can be found in the SI, Section 5 .

In Figure 2, we present the (a-c) real, $\varepsilon_{1}(E)$, and (d-f) imaginary, $\varepsilon_{2}(E)$, permittivity of the ITO, AZO and GZO films, respectively. We present the results, for the low- $N$ (dark colored lines) and high- $N$ (light colored lines) films, from fitting IR (red lines) and NIR-VIS-UV-SE (blue lines) measurements separately. The requirement for the two additional oscillators in the IR for ITO was previously noted by Uprety et al. ${ }^{34}$ They ascribed the peak at $0.045 \mathrm{eV}$ to Sn-O, Sn-O-Sn and In$\mathrm{O}$ optical phonon modes and a broader peak at $\sim 0.26 \mathrm{eV}$ to $\mathrm{Sn}-\mathrm{OH}$ and $\mathrm{O}-\mathrm{H}$ stretching, present due to water molecules within the chamber during the initial stage of deposition. However, the appearance of the broader peak at significantly lower energy $(\sim 0.13 \mathrm{eV})$ for this set of sputtered ITO films, alongside the observation that the energy of this peak changes with the films' carrier concentration (see SI, Section 5) suggests that the peak may instead be related to shallow defect 
states. For AZO and GZO, we again required two additional oscillators in the IR. The peak at $0.05 \mathrm{eV}$ is ascribed to a strong optical phonon absorption of $\mathrm{ZnO} .61,62$ However, we also note a large broad peak at $\sim 0.12 \mathrm{eV}$ which is not present for undoped $\mathrm{ZnO}$. Uprety et al. ${ }^{35}$ required two additional peaks at $0.11 \mathrm{eV}$ and $0.16 \mathrm{eV}$ to fit IR-SE measurements of AZO, which they attributed to phonon modes. However, to the best of our knowledge, correlation of these peaks to Raman spectra is not possible due to the limited spectral range of current Raman analysis of undopedand/or doped-ZnO. ${ }^{58,61-63}$ It is of great interest to determine whether this peak is due to additional phonon modes caused by the presence of substitutional dopants, phonon-plasmon coupling, ${ }^{64,65}$ defect-states or if it is an artefact arising from a non-uniform structure. ${ }^{66}$ Determination of the precise mechanism behind this absorption is, however, beyond the scope of this work.

In addition to the permittivity, for each film in Figure 2, we present only the Drude term that was determined via IR-SE (dashed black lines) alongside the extrapolation of the NIR-VIS-UV permittivity into the IR (dashed blue lines). To highlight the effect of NIR tailing, we indicate the region between the measured permittivity and the expected permittivity, if only free carriers were present, by the purple shaded region. We note that the NIR tailing is more significant for the doped $\mathrm{ZnO}$ films than for ITO, due to the increased intensity of the absorption peak at $\sim 0.12 \mathrm{eV}$. The increased tailing results in the higher value of $N_{\text {opt }}$ when fitting the doped $\mathrm{ZnO}$ simulated data sets in the NIR-VIS-UV that is observed in Figures 1d-f. This, subsequently, results in a larger limit for accurate extraction of the carrier transport properties as a higher free carrier absorption is required to screen the tailing of the IR absorption.

The high- $N$ ITO film is the only experimental material that shows no clearly visible NIR tailing and, therefore, IR and NIR-VIS-UV-SE agree to within 10\% (Figure 1d). For the doped ZnO materials, we note that the absorption peak at $\sim 0.05 \mathrm{eV}$ results in a region of positive real 
permittivity for lower energies. This prevents any exploitation of plasmonic behavior in the farIR. Conversely, for the low- $N$ ITO, AZO and GZO films, the additional absorption peaks in the IR result in a window (or two distinct windows) of negative permittivity which is not present when only the free carrier absorption is considered. Therefore, exploitation of plasmonic (or in this case phononic) behavior is possible despite the insufficient conductivity.

\section{FIGURES OF MERIT}

A key result of the developed understanding of the requirements for IR-SE is that we are able to confidently expand the work of Lalisse et al., ${ }^{67}$ Doirion et al. ${ }^{68}$ and others ${ }^{24,69-71}$ to accurately provide an array of useful figures of merit (FoMs) for key TCO material candidates with plasmonic responses in the IR. No plasmonic material is perfect and assessing the best material for each potential application is not trivial. To address this, the plasmonic community adopts universal dimensionless FoMs. ${ }^{31,37,70}$ Two widely used FoMs evaluate the quality of both localized and propagating SPP, which are calculated from the $\varepsilon_{1}(E)$ and $\varepsilon_{2}(E)$. Lallise et al. ${ }^{67}$ introduced two more useful FoMs, namely Faraday, $F a$, and Joule, Jo, numbers which quantify the ability of a nanoparticle to enhance the optical near field and produce heat, respectively. These FoMs take into account the refractive index of the local dielectric medium and light source by introducing or removing a $1 / n_{s}$ term in the $F a$ or $J o$ number, respectively. They drew up the values of the nonretarded resonance wavelength and $F a$ and $J o$ number at this wavelength for a large set of materials with plasmonic responses in the visible.

$$
\begin{gathered}
F a(E)=|1+2 \xi(E)|^{2} \\
J o(E)=\frac{e \varepsilon_{2}(E)}{n_{s}}|\xi(E)-1|^{2}
\end{gathered}
$$

where $n_{S}$ the refractive index of the surrounding medium and $\xi(E)$ is the enhancement factor, given by: 


$$
\xi(E)=\frac{\varepsilon_{1}(E)+i \varepsilon_{2}(E)-\varepsilon_{M}}{\varepsilon_{1}(E)+i \varepsilon_{2}(E)+2 \varepsilon_{M}}
$$

where $\varepsilon_{M}$ is the permittivity of the local dielectric medium.

Figure 3 presents the (a-c) Faraday and (e-g) Joule numbers for ITO, AZO and GZO, respectively, thin films produced for this investigation. We present the FoMs for the TCO films with a high- $N$ (black solid and green dashed lines) and low- $N$ (red solid and blue dashed lines). Here, we show both FoMs resulting from fitting SE measurements in the IR (solid lines), alongside the FoMs when extrapolating the optical constants fitting the NIR-VIS-UV spectra (dashed lines). For comparison, we also present the $F a$ and $J o$ numbers for $\mathrm{Ag}$ (black), $\mathrm{Al}$ (red), $\mathrm{Au}$ (blue) and TiN (green) in Figures $3 \mathrm{~d}$ and 3h, respectively. ${ }^{72,73}$ In addition, Table 2 lays out the values of $F a$ and $J o$ at resonance alongside the screened plasma energy $E_{p s}=E\left(\varepsilon_{1}=0\right)$.

Table 2. Near-field enhancement, $\mathrm{Fa}^{0}$, and photothermal conversion, $\mathrm{Jo}^{0}$, numerical figure of merits for the noble metals and sputtered films.

\begin{tabular}{|l|l|l|l|l|l|l|l|l|}
\hline \multirow{2}{*}{ Material } & \multicolumn{2}{|c|}{$E_{p s} / \lambda_{c o}$} & \multicolumn{2}{c|}{$\lambda_{\text {res }}^{F a}$} & $F a^{0}$ & \multicolumn{2}{c|}{$\lambda_{\text {res }}^{J o}$} & $J^{0}$ \\
\cline { 2 - 9 } & \multicolumn{1}{|c|}{$e V$} & $\mu m$ & $e V$ & $\mu m$ & & $e V$ & $\mu m$ & \\
\hline $\mathrm{Ag}$ & 4.74 & 0.262 & 3.45 & 0.359 & 107 & 3.49 & 0.355 & 49.9 \\
\hline $\mathrm{Al}$ & $>6.50$ & $<0.191$ & $>6.50$ & $<0.191$ & -- & $>6.50$ & $<0.191$ & -- \\
\hline $\mathrm{Au}$ & $>6.50$ & $<0.191$ & 2.35 & 0.528 & 17.8 & $>6.50$ & $<0.191$ & -- \\
\hline TiN & 2.56 & 0.484 & 2.12 & 0.585 & 13.4 & 2.42 & 0.512 & 5.26 \\
\hline ITO $_{\text {High } N}$ & 0.617 & 2.01 & 0.487 & 2.55 & 36.7 & 0.513 & 2.42 & 3.95 \\
\hline ITO $_{\text {Low } N}$ & 0.0761 & 16.3 & 0.0664 & 18.7 & 9.34 & 0.0728 & 17.0 & 0.0896 \\
\hline $\mathrm{AZO}_{\text {High } N}$ & 0.550 & 2.25 & 0.423 & 2.93 & 11.4 & 0.492 & 2.52 & 1.60 \\
\hline $\mathrm{AZO}_{\text {Low } N}$ & 0.359 & 3.45 & 0.281 & 4.41 & 8.14 & 0.359 & 3.45 & 0.740 \\
\hline $\mathrm{GZO}_{\text {High } N}$ & 0.663 & 1.87 & 0.505 & 2.46 & 17.9 & 0.557 & 2.23 & 2.65 \\
\hline
\end{tabular}


$\mathrm{Fa}$ and $\mathrm{Jo}$ numerical figure of merits are recorded at their respective resonance wavelengths, $\lambda_{\text {res }}^{F a}$ and $\lambda_{\text {res }}^{J o}$ in $\mathrm{eV}$ and $\mu \mathrm{m}$. Also shown are the screened plasma energy $(\mathrm{eV}) /$ crossover wavelength $(\mu m), E_{p s} / \lambda_{c o}$.

The Faraday number (Figure 3a-d) shows clear improvements of each TCO candidate for nearfield enhancement in the IR where $\mathrm{Au}$ and TiN are ineffective due to the large electronic losses at these photon energies. In fact, within the non-retarded regime considered by these FoMs, Au and TiN lack any plasmonic enhancement in the IR. Despite the additional oscillators for ITO, AZO and GZO having energy far below the resonance, the broad nature of these peaks strongly influences the Faraday number at resonance, resulting in a significant reduction in their predicted performance in comparison to that suggested from NIR-VIS-UV-SE alone. Specifically, by considering the IR during determination of the optical constants for the high- $N$ ITO, GZO and AZO films, the FoMs for near-field enhancement (relative to the static case where $F a=9$ ) are reduced by $9 \%, 74 \%$ and $20 \%$, respectively, from the NIR-VIS-UV result. For AZO and GZO, we find that the resonance energy is also blue-shifted due to the deviations revealed by IR-SE. For the low- $N$ AZO and GZO films, the detection of additional absorption centers revealed by IR-SE has a palpable effect in the $F a$ and $J o$ numbers (Figure 3 ). When considering IR-SE we reveal that ITO provides the strongest un-retarded near-field enhancement out of the three key TCO candidates, largely due to its higher mobility. We observe similar results for the Joule number in Figures 3e-h. We present the FoMs for SPP, LSPR and the Mie absorption efficiency in the SI, Section 6.

\section{CONCLUSIONS}

To conclude, we have investigated the limitations of NIR-VIS-UV-SE and IR-SE, independently, to determine the free carrier transport properties and optical constants of key TCO material candidates for IR plasmonics. At first, we fabricated a large set of ITO, AZO and GZO 
thin films and characterized their transport properties, via SE and Hall Effect, to establish the relationship between the effective mass, carrier concentration and mobility for each material due to non-parabolicity in the conduction band and various scattering mechanisms. Importantly, we brought another perspective to the physical mechanisms behind the large broad peak in the IR for ITO, AZO and GZO. Furthermore, we found that the presence of GBS in doped $\mathrm{ZnO}$ could diminish the free carrier mobility, and thus the plasmonic 'quality', when the defects are manipulated to red shift the plasma energy further into the IR.

We then utilized the established $\mu(N)$ and $m_{e}^{*}(N)$ relationships and determined, via ellipsometry, absorption centers to simulate a set of SE measurements covering a wide range of carrier concentrations for ITO, AZO and GZO. We demonstrated that there are three vital factors to consider clarifying when SE correctly determines the transport properties of low carrier concentration materials: NIR tailing, GBS and spectral sensitivity. By considering each case, we can build confidence in material properties extracted via SE. For the TCO candidates investigated, we confirmed that the phonon and/or defect state absorption in the IR, for each material, has significant tailing into the NIR. This results in an overestimation of the Drude contribution to the permittivity in the NIR-VIS-UV. The free carrier absorption dominates the spectrum in the NIR, potentially allowing for unambiguous determination of the carrier transport properties for carrier concentrations that fall outside material-dependent 'regions of uncertainty'. These regions are bounded by an adjustable 'acceptable deviation' of $N$ from the actual (simulated) value. We introduced the concept of the application-specificity for the region of uncertainty, allowing us to provide a confidence factor for NIR-VIS-UV-SE that can be applied on a case-by-case basis. For plasmonic applications, we use an acceptable deviation of $10 \%$. This identifies the region of uncertainty as $N \leq(3.0 \pm 0.5) \times 10^{19} \mathrm{~cm}^{-3}, N \leq(7 \pm 1) \times 10^{20} \mathrm{~cm}^{-3}$ and $N \leq(7 \pm 2) \times$ 
$10^{20} \mathrm{~cm}^{-3}$ for ITO, AZO and GZO, respectively. Within these regions, IR-SE measurements become of utmost importance. Moreover, we elucidated the more complex limits that are encountered by NIR-VIS-UV and IR-SE by considering inherent material limitations, measurement noise and the presence of a substrate, across a wide range of carrier concentrations. We reveal how the material structure always requires great consideration as both IR and NIR-VISUV-SE fail for materials where GBS is a dominant scattering mechanism. Vitally, the methodology outlined in this work can be applied to new materials to ensure confidence in measured and reported carrier transport properties determined via SE.

Finally, we demonstrated the importance of acquiring accurate measurements of the optical constants in the IR by expanding the library of FoMs for plasmonic applications to include the TCOs. We noted how measurements of the permittivity of TCOs via NIR-VIS-UV-SE overestimates their plasmonic quality. We identified that the additional absorption in the IR prevent the exploitation of plasmonic phenomena in the far-IR but create pockets of negative real permittivity for metal oxide films with low conductivity, allowing for exploitation of phononic behavior in the IR.

\section{ASSOCIATED CONTENT}

Supporting Information. Materials and methods, fitting strategy, evaluation of $m_{e}^{*}(N)$, further ellipsometric limits (random noise, presence of a substrate, thickness, additional oscillators, case for experimental films), raw and fit ellipsometric measurements, optical constants, fitting parameters and additional figures of merit. This material is available free of charge via the Internet at http://pubs.acs.org. 


\title{
AUTHOR INFORMATION
}

\section{Corresponding Author}

Nikolaos Kalfagiannis - Nottingham Trent University, School of Science and Technology, Nottingham, NG11 8NS, UK

Email: nikolaos.kalfagiannis@ntu.ac.uk

\section{Authors}

James A. Hillier - Nottingham Trent University, School of Science and Technology, Nottingham, NG11 8NS, UK

Sophie Camelio - Institut Pprime, UPR 3346 CNRS-Université de Poitiers, Poitiers, France

Wayne Cranton - Sheffield Hallam University, Materials and Engineering Research Institute, Sheffield, S1 1WB, UK

Alexei V. Nabok - Sheffield Hallam University, Materials and Engineering Research Institute, Sheffield, S1 1WB, UK

Christopher J. Mellor - The University of Nottingham, School of Physics and Astronomy, Nottingham, NG7 2RD, UK

Demosthenes C. Koutsogeorgis - Nottingham Trent University, School of Science and Technology, Nottingham, NG11 8NS, UK

\author{
Author Contributions \\ The manuscript was written through contributions of all authors. All authors have given approval \\ to the final version of the manuscript.
}




\title{
Funding Sources
}

James A. Hillier acknowledges funding from the Nottingham Trent University Vice Chancellor's PhD Bursary Scheme.

Nikolaos Kalfagiannis acknowledges support from Nottingham Trent University, School of Science and Technology capital equipment funding scheme for acquiring the Infrared Spectroscopic Ellipsometry system.

Sophie Camelio acknowledges the "Région Nouvelle-Aquitaine" and the European Regional Development Fund (ERDF 2014-2020) for funding within the IMATOP Project No. P-2016BAFE-209.Notes

\section{ACKNOWLEDGMENT}

The authors would like to acknowledge Joel Mohrmann, Ron Synowicki and Tom Tiwald from J. A. Woollam Co., Inc. for their insightful comments regarding this work.

\begin{abstract}
ABBREVIATIONS
SPP, surface plasmon polaritons; LSPR, localized surface plasmon resonances; CMOS, complementary metal-oxide-semiconductor; TCO, transparent conductive oxide; ITO, tin-doped indium oxide; AZO, aluminium-doped zinc oxide; GZO, gallium-doped zinc oxide; IR, infrared; THz, terahertz; SE, spectroscopic ellipsometry; NIR-VIS-UV, near-infrared-visible-ultraviolet; FoM, figures of merit; SI, supporting information; IIS, ionized impurity scattering; GBS, grain boundary scattering; IGZO, indium-gallium-zinc-oxide.
\end{abstract}




\section{REFERENCES}

(1) Atwater, H. A.; Polman, A. Plasmonics for Improved Photovoltaic Devices. Nat. Mater. 2010, 9 (3), 205-213.

(2) Brongersma, M. L.; Shalaev, V. M. The Case for Plasmonics. Science. 2010, pp 440-441.

(3) Schuller, J. A.; Barnard, E. S.; Cai, W.; Jun, Y. C.; White, J. S.; Brongersma, M. L. Plasmonics for Extreme Light Concentration and Manipulation. Nat. Mater. 2010, 9 (3), 193-204.

(4) Ozbay, E. Plasmonics: Merging Photonics and Electronics at Nanoscale Dimensions. Science. 2006, pp 189-193.

(5) Gramotnev, D. K.; Bozhevolnyi, S. I. Plasmonics beyond the Diffraction Limit. Nat. Photonics 2010, 4 (2), 83-91.

(6) Sun, K.; Xiao, W.; Ye, S.; Kalfagiannis, N.; Kiang, K. S.; Groot, C. H. (Kees.; Muskens, O. L. Embedded Metal Oxide Plasmonics Using Local Plasma Oxidation of AZO for Planar Metasurfaces. Adv. Mater. 2020, 2001534.

(7) Boriskina, S. V.; Ghasemi, H.; Chen, G. Plasmonic Materials for Energy: From Physics to Applications. Mater. Today 2013, 16 (10), 375-386.

(8) Boriskina, S. V.; Green, M. A.; Catchpole, K.; Yablonovitch, E.; Beard, M. C.; Okada, Y.; Lany, S.; Gershon, T.; Zakutayev, A.; Tahersima, M. H.; et al. Roadmap on Optical Energy Conversion. J. Opt. 2016, 18 (7), 73004.

(9) Homola, J.; Yee, S. S.; Gauglitz, G. Surface Plasmon Resonance Sensors: Review. Sensors Actuators, B Chem. 1999, 54 (1), 3-15.

(10) Mayer, K. M.; Hafner, J. H. Localized Surface Plasmon Resonance Sensors. Chem. Rev. 2011, 111 (6), 3828-3857. 
(11) Siozios, A.; Koutsogeorgis, D. C.; Lidorikis, E.; Dimitrakopulos, G. P.; Kehagias, T.; Zoubos, H.; Komninou, P.; Cranton, W. M.; Kosmidis, C.; Patsalas, P. Optical Encoding by Plasmon-Based Patterning: Hard and Inorganic Materials Become Photosensitive. Nano Lett. 2012, 12 (1), 259-263.

(12) Pincella, F.; Isozaki, K.; Miki, K. A Visible Light-Driven Plasmonic Photocatalyst. Light Sci. Appl. 2014, 3 (e113), 1-6.

(13) Martirez, J. M. P.; Carter, E. A. Prediction of a Low-Temperature N2 Dissociation Catalyst Exploiting near-IR-to-Visible Light Nanoplasmonics. Sci. Adv. 2017, 3 (12), 1-11.

(14) Zenin, V. A.; Choudhury, S.; Saha, S.; Shalaev, V. M.; Boltasseva, A.; Bozhevolnyi, S. I. Hybrid Plasmonic Waveguides Formed by Metal Coating of Dielectric Ridges. Opt. Express 2017, $25(11), 12295$.

(15) Lapotko, D. Plasmonic Nanobubbles as Tunable Cellular Probes for Cancer Theranostics. Cancers (Basel). 2011, 3 (1), 802-840.

(16) Huang, X.; El-Sayed, M. A. Plasmonic Photo-Thermal Therapy (PPTT). Alexandria J. Med. 2011, 47 (1), 1-9.

(17) Kim, S.; Kim, J. M.; Park, J. E.; Nam, J. M. Nonnoble-Metal-Based Plasmonic Nanomaterials: Recent Advances and Future Perspectives. Advanced Materials. 2018, pp 1-24.

(18) Boltasseva, A.; Atwater, H. A. Low-Loss Plasmonic Metamaterials. Science. 2011, 331 (6015), 290-291.

(19) Guler, U.; Kildishev, A. V.; Boltasseva, A.; Shalaev, V. M. Plasmonics on the Slope of Enlightenment: The Role of Transition Metal Nitrides. Faraday Discuss. 2015, 178, 71-86.

(20) Blaber, M. G.; Arnold, M. D.; Ford, M. J. A Review of the Optical Properties of Alloys and Intermetallics for Plasmonics. J. Phys. Condens. Matter 2010, 22 (14). 
(21) Fafin, A.; Yazidi, S.; Camelio, S.; Babonneau, D. Near-Field Optical Properties of AgxAu1-x Nanoparticle Chains Embedded in a Dielectric Matrix. Plasmonics 2016, 11 (5), $1407-$ 1416.

(22) Rajendra, R.; Bhatia, P.; Justin, A.; Sharma, S.; Ballav, N. Homogeneously-Alloyed GoldSilver Nanoparticles as per Feeding Moles. J. Phys. Chem. C 2015, 119 (10), 5604-5613.

(23) Peña-Rodríguez, O.; Caro, M.; Rivera, A.; Olivares, J.; Perlado, J. M.; Caro, A. Optical Properties of Au-Ag Alloys: An Ellipsometric Study. Opt. Mater. Express 2014, 4 (2), 403.

(24) Guler, U.; Shalaev, V. M.; Boltasseva, A. Nanoparticle Plasmonics: Going Practical with Transition Metal Nitrides. Mater. Today 2015, 18 (4), 227-237.

(25) Patsalas, P.; Kalfagiannis, N.; Kassavetis, S.; Abadias, G.; Bellas, D. V.; Lekka, C.; Lidorikis, E. Conductive Nitrides: Growth Principles, Optical and Electronic Properties, and Their Perspectives in Photonics and Plasmonics. Mater. Sci. Eng. R Reports 2018, 123, 1-55.

(26) Patsalas, P.; Kalfagiannis, N.; Kassavetis, S. Optical Properties and Plasmonic Performance of Titanium Nitride. Materials (Basel). 2015, 8 (6), 3128-3154.

(27) Babicheva, V. E.; Kinsey, N.; Naik, G. V.; Ferrera, M.; Lavrinenko, A. V.; Shalaev, V. M.; Boltasseva, A. Towards CMOS-Compatible Nanophotonics: Ultra-Compact Modulators Using Alternative Plasmonic Materials. Opt. Express 2013, 21 (22), 27326.

(28) Zhong, Y.; Malagari, S. D.; Hamilton, T.; Wasserman, D. Review of Mid-Infrared Plasmonic Materials. J. Nanophotonics 2015, 9 (1), 093791.

(29) Naik, G. V.; Shalaev, V. M.; Boltasseva, A. Alternative Plasmonic Materials: Beyond Gold and Silver. Adv. Mater. 2013, 25 (24), 3264-3294. 
(30) Caldwell, J. D.; Lindsay, L.; Giannini, V.; Vurgaftman, I.; Reinecke, T. L.; Maier, S. A.; Glembocki, O. J. Low-Loss, Infrared and Terahertz Nanophotonics Using Surface Phonon Polaritons. Nanophotonics 2015, 4 (1), 44-68.

(31) Wang, Y.; Overvig, A. C.; Shrestha, S.; Zhang, R.; Wang, R.; Yu, N.; Dal Negro, L. Tunability of Indium Tin Oxide Materials for Mid-Infrared Plasmonics Applications. Opt. Mater. Express 2017, 7 (8), 2727.

(32) Kanehara, M.; Koike, H.; Yoshinaga, T.; Teranishi, T. Indium Tin Oxide Nanoparticles with Compositionally Tunable Surface Plasmon Resonance Frequencies in the Near-IR Region. J. Am. Chem. Soc. 2009, 131 (49), 17736-17737.

(33) Babicheva, V. E.; Boltasseva, A.; Lavrinenko, A. V. Transparent Conducting Oxides for Electro-Optical Plasmonic Modulators. Nanophotonics 2015, 4 (1), 165-185.

(34) Uprety, P.; Junda, M. M.; Salmon, H.; Podraza, N. J. Understanding near Infrared Absorption in Tin Doped Indium Oxide Thin Films. J. Phys. D. Appl. Phys. 2018, 51 (29).

(35) Uprety, P.; Junda, M. M.; Ghimire, K.; Adhikari, D.; Grice, C. R.; Podraza, N. J. Spectroscopic Ellipsometry Determination of Optical and Electrical Properties of Aluminum Doped Zinc Oxide. Appl. Surf. Sci. 2017, 421, 852-858.

(36) Wang, T.; Zalkovskij, M.; Iwaszczuk, K.; Lavrinenko, A. V.; Naik, G. V.; Kim, J.; Boltasseva, A.; Jepsen, P. U. Ultrabroadband Terahertz Conductivity of Highly Doped ZnO and ITO. Opt. Mater. Express 2015, 5 (3), 566.

(37) Noginov, M. A.; Gu, L.; Livenere, J.; Zhu, G.; Pradhan, A. K.; Mundle, R.; Bahoura, M.; Barnakov, Y. A.; Podolskiy, V. A. Transparent Conductive Oxides: Plasmonic Materials for Telecom Wavelengths. Appl. Phys. Lett. 2011, 99 (2), 2-5. 
(38) De Nicolás, S. M.; Muñoz, D.; Denis, C.; Lerat, J. F.; Emeraud, T. Optimisation of ITO by Excimer Laser Annealing for A-Si:H/c-Si Solar Cells. Energy Procedia 2012, 27 (0), 586-591.

(39) El Hamali, S. O.; Cranton, W. M.; Kalfagiannis, N.; Hou, X.; Ranson, R.; Koutsogeorgis, D. C. Enhanced Electrical and Optical Properties of Room Temperature Deposited Aluminium Doped Zinc Oxide (AZO) Thin Films by Excimer Laser Annealing. Opt. Lasers Eng. 2016, 80, $45-51$.

(40) Liu, H.; Avrutin, V.; Izyumskaya, N.; Özgr, Ü.; Morkoç, H. Transparent Conducting Oxides for Electrode Applications in Light Emitting and Absorbing Devices. Superlattices Microstruct. 2010, 48 (5), 458-484.

(41) Exarhos, G. J.; Zhou, X. D. Discovery-Based Design of Transparent Conducting Oxide Films. Thin Solid Films 2007, 515 (18), 7025-7052.

(42) Romanyuk, V.; Dmitruk, N.; Karpyna, V.; Lashkarev, G.; Popovych, V.; Dranchuk, M.; Pietruszka, R.; Godlewski, M.; Dovbeshko, G.; Timofeeva, I.; et al. Optical and Electrical Properties of Highly Doped ZnO:Al Films Deposited by Atomic Layer Deposition on Si Substrates in Visible and near Infrared Region. Acta Phys. Pol. A 2016, 129 (1), A36-A40.

(43) Liu, X.; Park, J.; Kang, J. H.; Yuan, H.; Cui, Y.; Hwang, H. Y.; Brongersma, M. L. Quantification and Impact of Nonparabolicity of the Conduction Band of Indium Tin Oxide on Its Plasmonic Properties. Appl. Phys. Lett. 2014, 105 (18).

(44) Fujiwara, H.; Kondo, M. Effects of Carrier Concentration on the Dielectric Function of $\mathrm{ZnO}: \mathrm{Ga}$ and In 2O 3:Sn Studied by Spectroscopic Ellipsometry: Analysis of Free-Carrier and Band-Edge Absorption. Phys. Rev. B - Condens. Matter Mater. Phys. 2005, 71 (7), 1-10.

(45) Feneberg, M.; Nixdorf, J.; Lidig, C.; Goldhahn, R.; Galazka, Z.; Bierwagen, O.; Speck, J. S. Many-Electron Effects on the Dielectric Function of Cubic In2 O3: Effective Electron Mass, 
Band Nonparabolicity, Band Gap Renormalization, and Burstein-Moss Shift. Phys. Rev. B 2016, $93(4)$.

(46) De Sousa Meneses, D.; Malki, M.; Echegut, P. Structure and Lattice Dynamics of Binary Lead Silicate Glasses Investigated by Infrared Spectroscopy. J. Non. Cryst. Solids 2006, 352 (8), $769-776$.

(47) Ellmer, K.; Mientus, R. Carrier Transport in Polycrystalline Transparent Conductive Oxides: A Comparative Study of Zinc Oxide and Indium Oxide. Thin Solid Films 2008, 516 (14), $4620-4627$.

(48) Seto, J. Y. W. The Electrical Properties of Polycrystalline Silicon Films. J. Appl. Phys. 1975, $46(12), 5247-5254$.

(49) Masetti, G.; Severi, M.; Solmi, S. Modeling of Carrier Mobility Against Carrier Concentration in Arsenic-, Phosphorus-, and Boron-Doped Silicon. IEEE Trans. Electron Devices 1983, 30 (7), 764-769.

(50) Ellmer, K.; Mientus, R. Carrier Transport in Polycrystalline ITO and ZnO:Al II: The Influence of Grain Barriers and Boundaries. Thin Solid Films 2008, 516 (17), 5829-5835.

(51) Frischbier, M. V.; Wardenga, H. F.; Weidner, M.; Bierwagen, O.; Jia, J.; Shigesato, Y.; Klein, A. Influence of Dopant Species and Concentration on Grain Boundary Scattering in Degenerately Doped In2O3 Thin Films. Thin Solid Films 2016, 614, 62-68.

(52) Teixeira, V.; Cui, H. N.; Meng, L. J.; Fortunato, E.; Martins, R. Amorphous ITO Thin Films Prepared by DC Sputtering for Electrochromic Applications. Thin Solid Films 2002, 420$421,70-75$.

(53) Ginley, D. S. Handbook of Transparent Conductors; Ginley, D. S., Hosono, H., Paine, D. C., Eds.; Springer: New York, USA, 2011. 
(54) Sharma, V.; Singh, S.; Asokan, K.; Sachdev, K. A Study on $100 \mathrm{MeV} \mathrm{O7+} \mathrm{Irradiated}$ $\mathrm{SnO} 2 / \mathrm{Ag} / \mathrm{SnO} 2$ Multilayer as Transparent Electrode for Flat Panel Display Application. Nucl. Instruments Methods Phys. Res. Sect. B Beam Interact. with Mater. Atoms 2016, 379, 141-145.

(55) Ellmer, K. Past Achievements and Future Challenges in the Development of Optically Transparent Electrodes. Nat. Photonics 2012, 6 (12), 809-817.

(56) Kim, H.; Osofsky, M.; Prokes, S. M.; Glembocki, O. J.; Piqué, A. Optimization of AlDoped ZnO Films for Low Loss Plasmonic Materials at Telecommunication Wavelengths. Appl. Phys. Lett. 2013, 102 (17), 1-5.

(57) Kim, J.; Naik, G. V.; Emani, N. K.; Guler, U.; Boltasseva, A. Plasmonic Resonances in Nanostructured Transparent Conducting Oxide Films. IEEE J. Sel. Top. Quantum Electron. 2013, $19(3)$.

(58) Zheng, H.; Zhang, R. J.; Li, D. H.; Chen, X.; Wang, S. Y.; Zheng, Y. X.; Li, M. J.; Hu, Z. G.; Dai, N.; Chen, L. Y. Optical Properties of Al-Doped ZnO Films in the Infrared Region and Their Absorption Applications. Nanoscale Res. Lett. 2018, 13.

(59) Xiao, S.; Drachev, V. P.; Kildishev, A. V.; Ni, X.; Chettiar, U. K.; Yuan, H. K.; Shalaev, V. M. Loss-Free and Active Optical Negative-Index Metamaterials. Nature 2010, 466 (7307), 735738.

(60) Naik, G. V.; Liu, J.; Kildishev, A. V.; Shalaev, V. M.; Boltasseva, A. Demonstration of $\mathrm{Al}: \mathrm{ZnO}$ as a Plasmonic Component for near-Infrared Metamaterials. Proc. Natl. Acad. Sci. U. S. A. 2012, $109(23), 8834-8838$.

(61) Huang, Y.; Liu, M.; Li, Z.; Zeng, Y.; Liu, S. Raman Spectroscopy Study of ZnO-Based Ceramic Films Fabricated by Novel Sol-Gel Process. Mater. Sci. Eng. B Solid-State Mater. Adv. Technol. 2003, 97 (2), 111-116. 
(62) Bundesmann, C.; Ashkenov, N.; Schubert, M.; Spemann, D.; Butz, T.; Kaidashev, E. M.; Lorenz, M.; Grundmann, M. Raman Scattering in ZnO Thin Films Doped with Fe, Sb, Al, Ga, and Li. Appl. Phys. Lett. 2003, 83 (10), 1974-1976.

(63) Ashkenov, N.; Mbenkum, B. N.; Bundesmann, C.; Riede, V.; Lorenz, M.; Spemann, D.; Kaidashev, E. M.; Kasic, A.; Schubert, M.; Grundmann, M.; et al. Infrared Dielectric Functions and Phonon Modes of High-Quality ZnO Films. J. Appl. Phys. 2003, 93 (1), 126-133.

(64) Zollner, S.; Paradis, P. P.; Abadizaman, F.; Samarasingha, N. S. Drude and Kukharskii Mobility of Doped Semiconductors Extracted from Fourier-Transform Infrared Ellipsometry Spectra. J. Vac. Sci. Technol. B 2019, 37 (1), 012904.

(65) Kukharskii, A. A. Plasmon-Phonon Coupling in GaAs. Solid State Commun. 1973, 13 (11), $1761-1765$.

(66) Özgür, Ü.; Alivov, Y. I.; Liu, C.; Teke, A.; Reshchikov, M. A.; Doğan, S.; Avrutin, V.; Cho, S. J.; Morko, H. A Comprehensive Review of ZnO Materials and Devices. J. Appl. Phys. 2005, 98 (4), 1-103.

(67) Lalisse, A.; Tessier, G.; Plain, J.; Baffou, G. Quantifying the Efficiency of Plasmonic Materials for Near-Field Enhancement and Photothermal Conversion. J. Phys. Chem. C 2015, 119 (45), 25518-25528.

(68) Doiron, B.; Mota, M.; Wells, M. P.; Bower, R.; Mihai, A.; Li, Y.; Cohen, L. F.; Alford, N. M. N.; Petrov, P. K.; Oulton, R. F.; et al. Quantifying Figures of Merit for Localized Surface Plasmon Resonance Applications: A Materials Survey. ACS Photonics. 2019, pp 240-259.

(69) Khurgin, J. B. Relative Merits of Phononics vs. Plasmonics: The Energy Balance Approach. Nanophotonics 2018, 7 (1), 305-316. 
(70) Berini, P. Figures of Merit for Surface Plasmon Waveguides. Opt. Express 2006, 14 (26), 13030.

(71) Manjavacas, A.; Liu, J. G.; Kulkarni, V.; Nordlander, P. Plasmon-Induced Hot Carriers in Metallic Nanoparticles. ACS Nano 2014, 8 (8), 7630-7638.

(72) Palik, E. D. Handbook of Optical Constants of Solids; Academic Press: San Diego, CA, 2012; Vol. 1.

(73) Patsalas, P.; Logothetidis, S. Optical, Electronic, and Transport Properties of Nanocrystalline Titanium Nitride Thin Films. J. Appl. Phys. 2001, 90 (9), 4725-4734. 


\section{LIST OF FIGURE CAPTIONS}

Figure 1. (a-c) Carrier concentration and mobility of sputtered ITO, AZO and GZO films, respectively, as determined by IR-SE (green circles) and Hall Effect (yellow squares). The fit of $\mu$ ( $N$ ) (eqs 2-7) to the Hall Effect measurements is shown by the black solid line. We highlight the regions where different scattering mechanisms are dominant with the different colors. (d-f) 'Optical' carrier concentration, $N_{\text {opt }}$, as determined by fitting each simulated dataset of $N_{\text {sim }}$ for ITO, AZO and GZO, respectively. We show the result from fitting the data simulated in the IR (red circles) and in the NIR-VIS-UV (blue squares) alongside $N_{\text {opt }}=N_{\text {sim }}$ (black dashed diagonal line). The influence of the NIR tailing and GBS are indicated by the purple and yellow shaded areas, respectively.

Figure 2. (a-c) Real, $\varepsilon_{1}(E)$, and (d-f) imaginary, $\varepsilon_{2}(E)$, parts of the complex permittivity for the sputtered ITO, AZO and GZO films, respectively, as fit in the IR (solid red lines), as fit in the NIRVIS-UV (solid blue lines), via extrapolation of the NIR-VIS-UV into the IR (dashed blue lines), and as simulated with only the Drude term of the IR fitted (dashed black/grey lines). 'Low-N' (darker lines) and 'High-N' (lighter lines) represent two distinct films of each material that cover the experimental range of carrier concentration. The purple shaded region highlights the difference between the simulated and fitted Drude contribution in the NIR, where tailing effects of the IR absorption centers are mistaken for free carrier absorption when fitting in the NIR-VIS-UV. Please note the changes in scale at -17 and 7 for the real permittivity and at 3 for the imaginary permittivity. 
Figure 3. (a-d) Faraday and (e-h) Joule numbers for ITO, AZO and GZO, respectively, alongside (d,h) Ag (black), Al (red), Au (blue) and TiN (green) for comparison. For the TCOs, we present FoMs for the high- $N$ (black solid and green dashed lines) and low- $N$ (red solid and blue dashed lines) films. The solid lines represent the 'true' FoM (calculated by optical constants derived by IR-SE) taking into account phonon and interband transitions and the dashed lines are the calculations employing extrapolated optical constants from NIR-VIS-UV-SE. Please note the logarithmic scales and the change in scale in the Faraday number at 10. 


\title{
For Table of Contents Only
}

\section{When Ellipsometry Works Best - A Case Study}

\section{With Transparent Conductive Oxides.}

\author{
James A. Hillier, Sophie Camelio, Wayne Cranton, Alexei V. Nabok, Christopher J. Mellor,
} Demosthenes C. Koutsogeorgis, and Nikolaos Kalfagiannis ${ }^{*}$

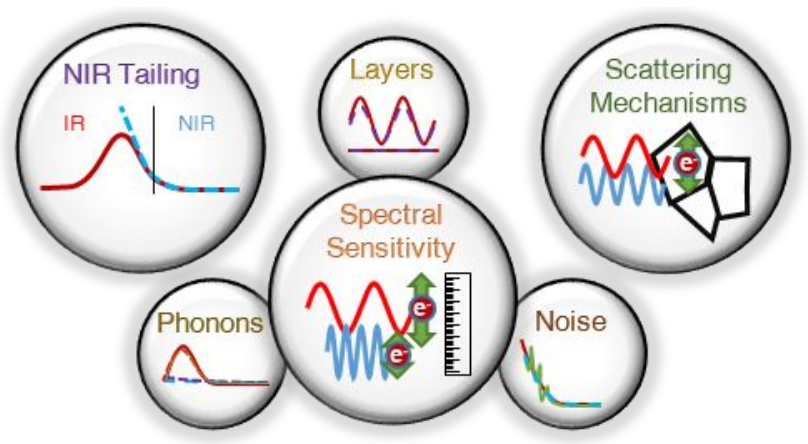

The graphic represents the different cases that are essential to consider when concerning the limitations of ellipsometry to determine the parameterized oscillators defining the optical constants of materials. 